\title{
Urinary System Disorder
}

National Cancer Institute

\section{Source}

National Cancer Institute. Urinary System Disorder. NCI Thesaurus. Code C3430.

Disorders of any part of the urologic system. 